\title{
Long-term results of long segment coronary artery lesions overlapped with novolimus-eluting DESolve scaffold: Disappointment or futuristic?
}

\author{
Ersin Ibişoğlu (D, Sinem Çakal¹ (1), Beytullah Çakal² (D), H. Murat Güneş² (D), \\ Bedrettin Boyraz ${ }^{3}$ (D) Bilal Boztosun² (D) \\ Department of Cardiology, Başakşehir Çam and Sakura City Hospital; İstanbul-Turkey \\ ${ }^{1}$ Department of Cardiology, İstanbul Haseki Training and Research Hospital; İstanbul-Turkey \\ ${ }^{2}$ Department of Cardiology, İstanbul Medipol University; İstanbul-Turkey \\ ${ }^{3}$ Department of Cardiology, Tatvan State Hospital; Bitlis-Turkey
}

\section{ABSTRACT}

Objective: The data on using novolimus-eluting DESolve bioresorbable scaffolds (BVS) for long-segment coronary artery lesions remains insufficient. In this study, our main objective was to assess the long-term effects of the overlapping applications of both DESolve-DESolve and the drug-eluting stent (DES)-DESolve.

Methods: A single-centered study of 103 patients scheduled for DESolve placement for long-segment lesions ( $>28 \mathrm{~mm}$ ) was conducted ( 0 ctober 2013 to November 2016). A DESolve-DESolve overlap was used on 43 patients and a DES-DESolve overlap on 60 patients. Acute procedural success and major adverse cardiac events (MACE) (stent thrombosis, targeted vessel revascularization, targeted lesion revascularization, and cardiac death) were evaluated. The patients were followed up for 48 months.

Results: Revascularization was performed on $4(6.7 \%)$ patients in the DES-DESolve group and $5(11.6 \%)$ patients in the DESolve-DESolve group for target lesion revascularization. Among the study population, $10(9.7 \%)$ patients had MACE, including $5(8.3 \%)$ patients in the DES-DESolve group and $5(11.6 \%)$ patients in the DESolve-DESolve group.

Conclusion: The positive results of our study concerning the use of DESolve for the treatment of long coronary lesions demonstrate that BVS will emerge with new platforms and become non-inferior to the DES.

Keywords: bioresorbable vascular scaffold, DESolve, overlapping, hybrid strategy

Cite this article as: Ibişoğlu E, Çakal S, Çakal B, Güneş HM, Boyraz B, Boztosun B. Long-term results of long segment coronary artery lesions overlapped with novolimus-eluting DESolve scaffold: Disappointment or futuristic? Anatol J Cardiol 2021; 25: 912-9.

\section{Introduction}

As studies reported in the most recent clinical settings, overlapping stents are available to approximately $30 \%$ of patients who undergo percutaneous coronary intervention (PCI) because of long coronary lesions in which the placement of various stents or dissection after implanting the previous stent is required (1). The overlapping application of first-generation DES is still related to compromised angiographic and clinical complications, such as myocardial infarction (MI) and death. However, according to second-generation DES results, the overlapping implantation of these stents is reliable and has relatively fewer risks for vessel revascularization or bad clinical outcomes $(2,3)$.

Bioresorbable vascular scaffolds (BVS) have been designed to address drug-eluting, stent-related biocompatibility concerns with progressive degradation (4). Treating coronary lesions with- 


\section{HIGHLIGHTS}

- Although it is not recommended to use evorulimuseluting biovascular scaffold (BVS) in practice owing to its long-term results, not all BVS should be evaluated in a similar manner. Even the positive results of DESolve in our study with long coronary lesions make it inevitable that BVS will emerge with new platforms and become non-inferior to DES.

out irreversible scaffolding and recovering receptive vasomotion would not limit any possible potential surgical revascularization (5). Reports have revealed better success and safety results restricted to straightforward, short, and stable lesions despite previous high expectations for BVS (6).

The novolimus eluting DESolve BVS (Elixir Medical Company, Sunnyvale, CA, USA) is made of poly-L lactide polymer scaffold covered with proliferative-resistant and anti-inflammatory agents. The biodegradation of the scaffold occurs over a year with 2-year full absorption, and the in vitro test has demonstrated the capability to provide the required radial power to support the vessel for a critical duration of 3 to 4 months following implantation (7).

The clinical effects of the overlapping of DES-DESolve and DESolve-DESolve in long segment coronary lesions were assessed in our study.

\section{Methods}

This study meets the guidelines of the Helsinki Declaration. The study procedure was approved by the National Medical Ethics Committee, and written informed consent was given by all the patients.

\section{Study population}

We conducted this retrospective, single-center, and nonrandomized study in the Medipol University, Department of Cardiology, Istanbul, Turkey. In this analysis, 103 patients who underwent PCI for long-segment lesions $(>28 \mathrm{~mm})$ that could not be protected with a single stent were registered at our clinic between October 2013 and November 2016. Overlapping with DESolve-DESolve or DES-DESolve was performed in these patients. The patients were treated with DESolve and everolimus-eluting DES (XIENCE PRO, Abbott Vascular, Santa Clara, CA, USA). There were specified fundamental clinical features, angiographic variables, and procedural features. Informed consent was obtained from all the patients before the procedure.

All the patients with stable coronary heart disease and acute coronary syndrome, except acute ST-segment elevation MI were enrolled. Patients admitted with stable angina pectoris were determined on the basis of the presence of chest pain at rest or on exertion with one positive finding from the exercise test and myocardial perfusion imaging. All coronary artery lesions had $>70 \%$ stenosis, were $>28 \mathrm{~mm}$ in length, and had a reference vessel diameter (RVD) of $>2.5 \mathrm{~mm}$. The primary exclusion criteria were left ventricular ejection fraction below $35 \%$, lesions found in the left main coronary artery, arterial or saphenous vein graft lesions, acute ST-elevation MI, and lesions expanding to the coronary ostium.

Once the patients were registered, follow-up results from outpatient clinic visits or emergency room admissions were collected, and patient surveillance was carried out by routine telephone calls. Information regarding control angiography and $\mathrm{PCI}$ was reported when the patients decided to undergo coronary angiography or $\mathrm{PCl}$ at a follow-up appointment or upon emergency room admission. The patients were followed up for 48 months.

\section{Procedures and medications}

The procedures were completed in compliance with the current $\mathrm{PCI}$ requirements. During treatment, all the patients were administered an upstream dose of $300 \mathrm{mg}$ acetylsalicylic acid (ASA) and a loading dose of 300 to $600 \mathrm{mg}$ clopidogrel, $180 \mathrm{mg}$ ticagrelor, or $60 \mathrm{mg}$ prasugrel. During the operation, all the patients were given unfractionated heparin $(100 \mathrm{U} / \mathrm{kg})$ as an anticoagulant, and an additional bolus was sometimes administered to obtain an active clotting period of 250 . None of the patients were administered glycoprotein IIb/IIla inhibitors.

Overlapping BVS distal to the DES was preferred if there were special lesion characteristics of calcification, tortuosity, or bifurcation and in prognostically significant segments and vessels, such as the left anterior descending artery, to enable future surgical vessel grafting options. Moreover, as BVS would lay on the top of the metallic struts at the overlapped junction, this would prevent probable metallic stent strut disruption and malapposition at the overlapped junction during temporary scaffold full resorption. In addition, the implantation times of BVSs are kept short; and therefore, it is suggested that it should be implanted as soon as possible and BVS should not be used when a certain period is exceeded. As the scaffold thickness is high in BVS, it can be difficult to pass through the DES, and this may cause a waste of time.

Scaffold implantation was accomplished with a constant pressure increase ( $1 \mathrm{~atm}$ increase per $5 \mathrm{~s}$ ), which was not over the rated burst pressure. Pre-dilatation was done before all the interventions. After DESolve implantation at the distal site, overlapping proximal DESolve or DES was performed on all the patients. The stent overlap was described as a $1 \mathrm{~mm}$ overlap of 2 stents angiographically (Fig. 1). Post-dilatation with a DESolve diameter not exceeding $0.5 \mathrm{~mm}$ was conducted on all the patients in the stent overlapping area. Post dilation of BVS with non-compliant balloons not exceeding $0.5 \mathrm{~mm}$ of the scaffold diameter at $18 \mathrm{~atm}$, whereas post-dilation of DES at $20 \mathrm{~atm}$ with non-compliant balloons of diameter up to $1.5 \mathrm{~mm}$ exceeding the stent diameter was performed. 

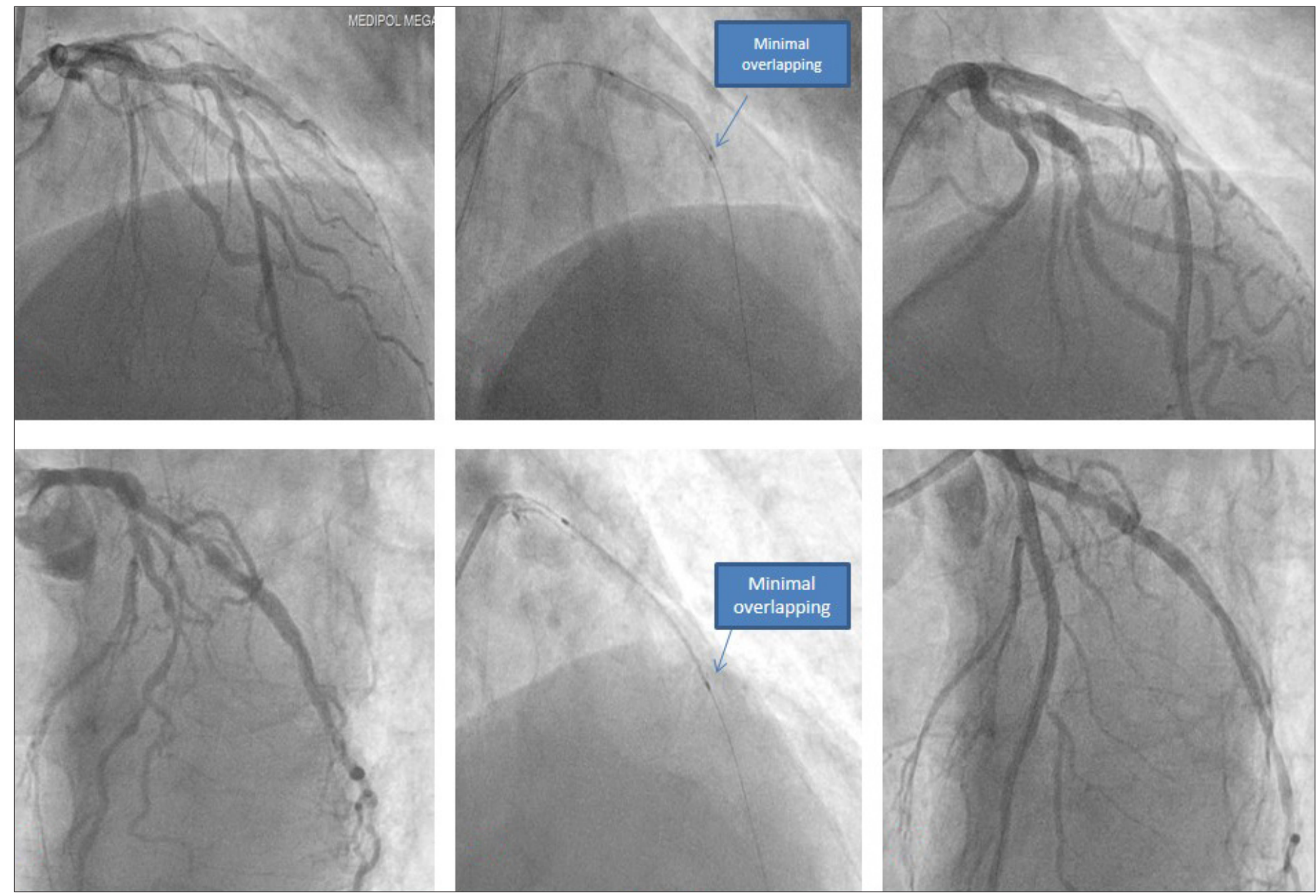

Figure 1. Angiographic method

\section{Angiographic criteria and quantitative coronary angiographic analysis}

Tortuosity has been described as showing a proximity $>45^{\circ}$ to the lesion. Mild tortuosity was classified as only 1 bending between $45^{\circ}$ and $90^{\circ}$ proximity to the lesion, whereas 3 or more bendings between $45^{\circ}$ and $90^{\circ}$ or 1 or several bendings $>90^{\circ}$ were defined as extreme tortuosity. Bending was classified as moderate tortuosity on the basis of these parameters (mild and extreme tortuosity) (8).

The calcifying of the vessel wall through the lesion location is known as open radiopacity. It was rated as mild (radiopacity was only observed throughout the cardiac cycle before the injected contrast) and extreme (radiopacity was found on either side of the vessel wall ahead of the contrast injection and apart from cardiac motion) (9).

Quantitative coronary angiography (OCA) was measured in one central angiographic laboratory using CAAS 5.9 (Pie Medical Imaging) in the angiographic research unit of the hospital. Standard and post-surgical minimum lumen diameter (MLD), mean lumen diameter (MnLD), RVD, residual diameter stenosis (DS\%), lesion diameter, and acute gain were evaluated. Angiographic data was measured with contrast-filled normal validation. Using similar projected angiography, these pictures were processed to eliminate dramatic shortening. Measurements were made separately by 2 blinded cardiologists. For assessments, the intra- and interobserver variations were $<5 \%$.

\section{Study endpoints and definitions}

Acute procedural success was defined as a final angiographic residual stenosis of $<30 \%$ with thrombolysis in myocardial infarction grade 3 flow. In-stent restenosis was described as a decrease in the DS\% of $>50 \%$ in the fragment in which the stent was placed.

MACE involving cardiac death, myocardial infarction (MI), target vessel revascularization (TVR), target lesion revascularization (TLR), and stent thrombosis comprised the final stage of this study.

The minimal luminal diameter of the lesion segment was described as MLD, and the maximum lumen diameter of the lesion segment was described as MnLD. Inside the index treatment stent or the $5 \mathrm{~mm}$ edge, TLR was classified as repeated PCI or procedural revascularization, whereas TVR was described as a revascularization operation that occurs beyond the edges of the stent or $5 \mathrm{~mm}$ inside the processed vessel. 


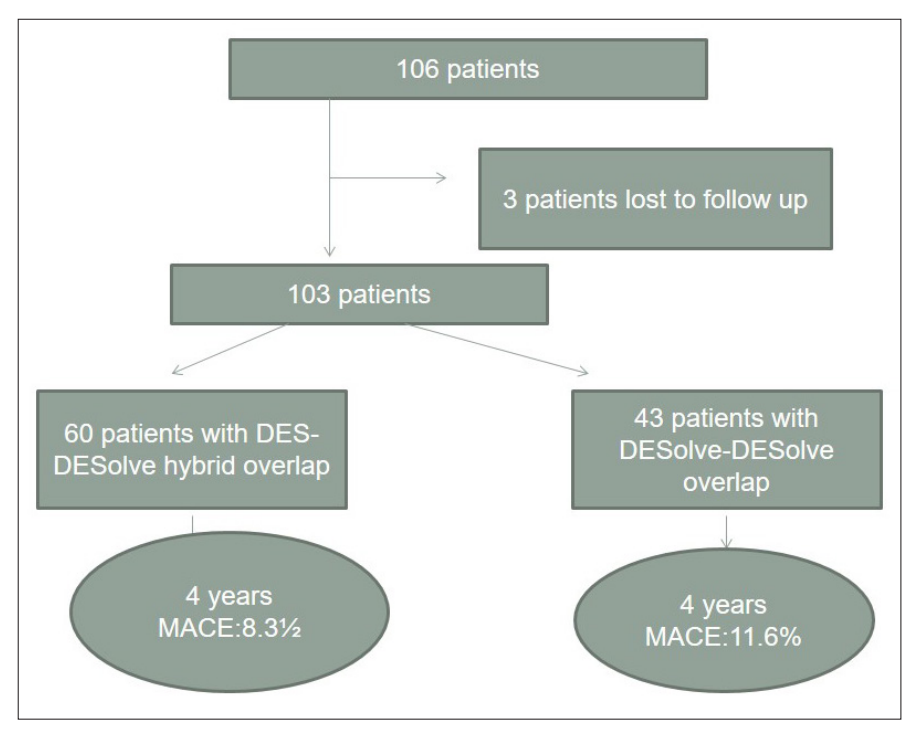

Figure 2. Patient population

\begin{tabular}{|lc|}
\hline \multicolumn{2}{|l|}{ Table 1. Baseline clinical characteristics of the patients } \\
\hline Age, years, & $56.73 \pm 8.5$ \\
Male sex, $\mathrm{n}(\%)$ & $93(90.3)$ \\
Hypertension, $\mathrm{n}(\%)$ & $57(55.3)$ \\
Diabetes mellitus, $\mathrm{n}(\%)$ & $38(36.9)$ \\
Current smoker, $\mathrm{n}(\%)$ & $49(47.6)$ \\
Dyslipidemia, $\mathrm{n}(\%)$ & $70(68)$ \\
Family history, $\mathrm{n}(\%)$ & $40(38.8)$ \\
Previous MI, $\mathrm{n}(\%)$ & $28(27.2)$ \\
Stable angina, $\mathrm{n}(\%)$ & $83(80.6)$ \\
Unstable angina, $\mathrm{n}(\%)$ & $15(14.6)$ \\
Heart failure, $\mathrm{n}(\%)$, & $4(3.9)$ \\
LVEF (\%), & $56.61 \pm 6.9$ \\
Hemoglobin (g/dL), & $14.3 \pm 8.6$ \\
Creatinine & $1.08 \pm 1.09$ \\
Platelets & $264.77 \pm 127.08$ \\
New P2Y & inhibitor use (n \%) \\
\hline LVEF - left ventricular ejection fraction; Ml - myocardial infarction \\
\hline
\end{tabular}

MI was described as an increase in cardiac troponin levels $\left(>5 \times 99^{\text {th }}\right.$ percentile) with ischemia indications, electrocardiography (ECG) modifications before the procedure, an increase of $>99^{\text {th }}$ percentile after the procedure, or the echocardiographic modifications suggesting ischemia.

\section{Statistical analysis}

Statistical analysis was conducted using the Statistical Package for the Social Sciences version 23.0 statistical tools (SPSS Inc., Chicago, IL, USA). Continuous variables have been presented as mean \pm standard deviation or, if applicable, medians and interquartile scale. Categorical values were presented as percentages. To measure the normality of the continuous variable distribution, the Kolmogorov-Smirnov test was used. To compare the mean of the group for continuous variables, the Student's t-test, the Mann-Whitney U test, the Kruskal-Wallis test, or analysis of variance was used when applicable.

To compare the categorical variables, the chi-squared test was applied. Descriptive statistical analysis for MACE was given as percentages and the minimum, maximum, and median monitoring periods of the patients. To evaluate the impact of DESolve-DESolve versus DES-DESolve overlapping on the outcome of TLR throughout the monitoring, Kaplan-Meier curves were created. The data were presented as log-rank, and p values were shown in graphs. A $p$-value of $\leq 0.05$ was considered statistically significant.

\section{Results}

The 103 patients enrolled in the study had 103 lesions that were subjected to overlapping. Follow-up data could not be obtained in 3 patients (e.g., a declined, asylum seeker) so they were excluded from the analysis (Fig. 2). The mean age of the patients was $56.73 \pm 8.5$ years, and $93(90.3 \%)$ of the patients were men. Risk factor assessment showed that $57(55.3 \%)$ patients had hypertension, and $38(36.9 \%)$ patients had diabetes mellitus. Furthermore, 70 $(68 \%)$ patients had dyslipidemia, and $49(47.6 \%)$ patients were active smokers. Both dyslipidemia and smoking were found to be important risk factors among the study group. Fifteen patients $(16.5 \%)$ who had unstable angina pectoris received $\mathrm{PCl}$. The mean left ventricular ejection fraction was $56.61 \pm 6.9 \mathrm{~mL} / \mathrm{min}$ (Table 1).

No significant differences in demographic characteristics between study groups with DES-DESolve hybrid overlapping and DESolve-DESolve overlapping were observed in this study (Table 2). In $81(78.6 \%)$ patients, femoral access was preferred and in 45 (43.7\%) patients, new P2Y12 inhibitors were favored in combination with ASA for dual antiplatelet therapy. New P2Y12 inhibitors were employed for $33(55 \%)$ patients in the DESDESolve group and $28(65.1 \%)$ in the DESolve-DESolve group.

Primary angiographic characteristics, for example, the type of lesions, the length of lesions, severe calcification, severe tortuosity, and treated vessels were found to be correlated with the DES-DESolve and DESolve-DESolve groups. Nonetheless, a significant difference between the DES-DESolve group (26.87 $\mathrm{mm} \pm 3.7 \mathrm{~mm})$ and the DESolve-DESolve group $(25.67 \mathrm{~mm} \pm 5.04$ $\mathrm{mm})(\mathrm{p}=0.048)$ was detected in terms of the length of the implanted stents. Distal DESolve implantation was performed in both the groups. There was no difference detected in the diameters of distal DESolves between the DES-BVS $(2.90 \pm 0.4)$ and the DESolve-DESolve $(2.98 \pm 0.4)$ groups $(p=0.577)$. All the patients underwent pre-dilatation and post-dilatation, and the percutaneous transluminal coronary angioplasty (PTCA) balloon size was found to be similar in the two groups (Table 3).

Two $(2.1 \%)$ patients exhibited procedural complications. One patient, who was managed by prolonged inflation of the balloon, underwent coronary rupture after high pressure post-dilatation 


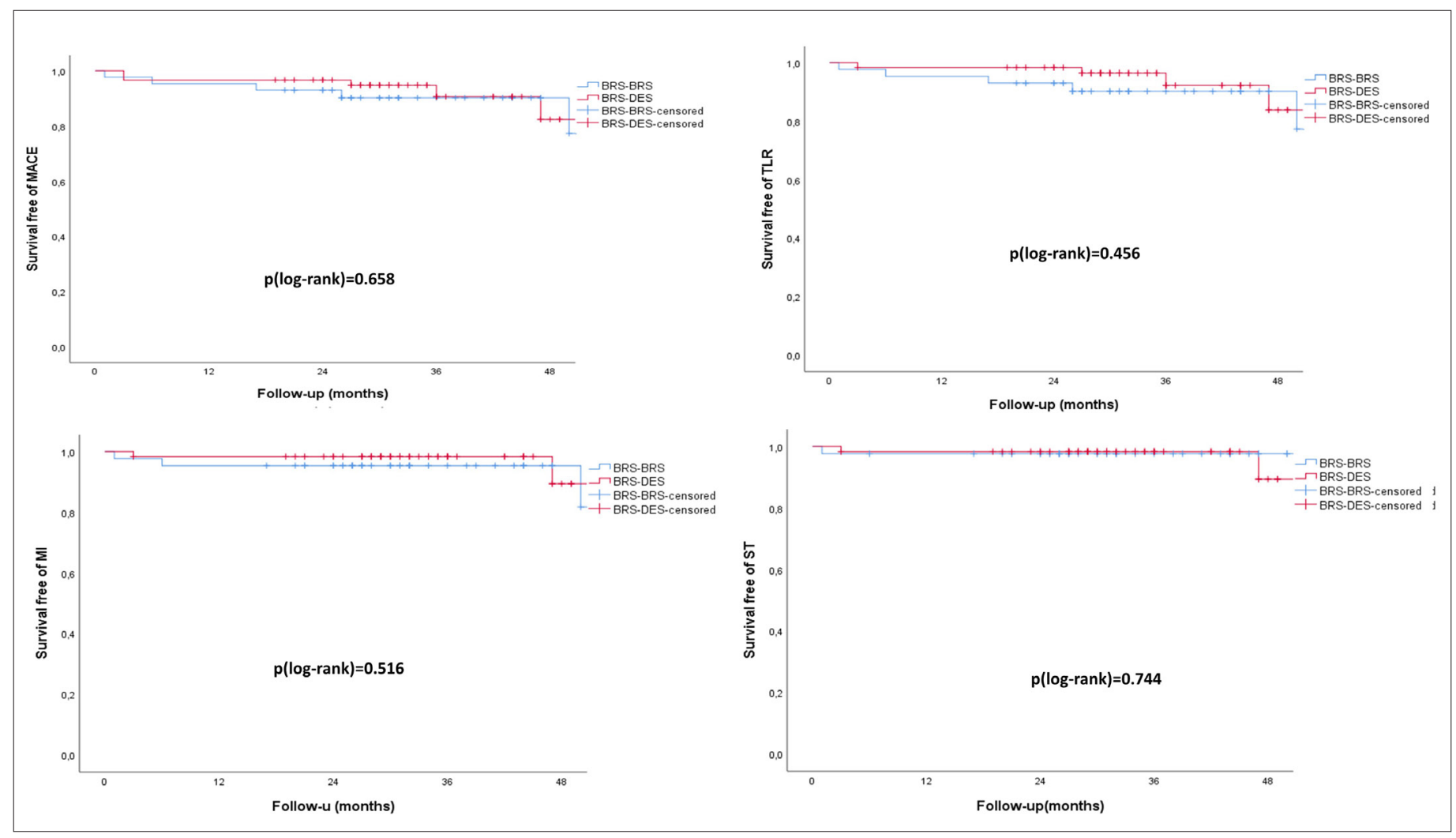

Figure 3. Major adverse cardiac event graphic

\begin{tabular}{|c|c|c|c|}
\hline Variables & $\begin{array}{l}\begin{array}{l}\text { DESolve-DESolve } \\
n=43\end{array} \\
\text { n }\end{array}$ & $\begin{array}{l}\text { DES-DESolve } \\
n=60\end{array}$ & $P$-value \\
\hline Age, years & $56.70 \pm 9.020$ & $56.75 \pm 8.23$ & 0.508 \\
\hline Male sex, $\mathrm{n}(\%)$ & $39(90.7 \%)$ & $53(88.3 \%)$ & 0.702 \\
\hline Hypertension, n (\%) & $25(58.1 \%)$ & $32(56.1 \%)$ & 0.628 \\
\hline $\begin{array}{l}\text { Diabetes mellitus, } \\
\mathrm{n}(\%)\end{array}$ & $18(41.9 \%)$ & $20(33.3 \%)$ & 0.376 \\
\hline Dyslipidemia, n (\%) & $31(44.3 \%)$ & $39(55.7 \%)$ & 0.069 \\
\hline Current smoker, n (\%) & $20(46.5 \%)$ & $29(48.3 \%)$ & 0.855 \\
\hline Family history, $\mathrm{n}(\%)$ & $19(44.2 \%)$ & $21(\% 35)$ & 0.346 \\
\hline Previous MI, n (\%) & $12(27.9 \%)$ & $16(26.7 \%)$ & 0.889 \\
\hline Stable angina, $\mathrm{n}(\%)$ & $38(88.4 \%)$ & $45(75 \%)$ & 0.091 \\
\hline Unstable angina, n (\%) & $5(11.6 \%)$ & $10(16.7 \%)$ & 0.475 \\
\hline Heart failure, n (\%) & $3(7 \%)$ & $1(1.7 \%)$ & 0.169 \\
\hline LVEF & $57 \pm 8.2$ & $56.33 \pm 5.7$ & 0.109 \\
\hline Hemoglobin & $13.65 \pm 2.03$ & $14.72 \pm 11.15$ & 0.305 \\
\hline Platelets & $279 \pm 179$ & $254 \pm 69$ & 0.081 \\
\hline Creatinine & $0.92 \pm 0.14$ & $1.2 \pm 1.4$ & 0.018 \\
\hline $\begin{array}{l}\text { New } \mathrm{P}_{2} \mathrm{Y}_{12} \text { inhibitor } \\
\text { use, } \mathrm{n}(\%)\end{array}$ & $28(65.1 \%)$ & $33(55 \%)$ & 0.303 \\
\hline \multicolumn{4}{|c|}{$\begin{array}{l}\text { DES - drug eluting stent; LVEF - left ventricular ejection fraction; MI - myocardial } \\
\text { infarction }\end{array}$} \\
\hline
\end{tabular}

owing to insufficient expansion of the calcified overlap site formation. In the other patient, the cerebrovascular event that recovered without sequela occurred during the post-procedural follow-up period in the hospital.

Neither patient required re-intervention, experienced adverse cardiac conditions, or died during the in-hospital stay period. Patient follow-up was carried out using a standard procedure for an average of 48 months either by direct clinical examination or by phone call during the 1 st $, 6^{\text {th }}, 12^{\text {th }}, 24^{\text {th }}, 36^{\text {th }}$, and $48^{\text {th }}$ months.

Figure 3 describes the cumulative incidence of survival-free event curves of MACE, TV-MI, ST, and TLR in both the groups. Of the study population, $10(9.7 \%)$ patients experienced MACE with no cardiovascular-related deaths, $5(8.3 \%)$ from the DESDESolve group and $5(11.6 \%)$ from the DESolve-DESolve group ( $p=0.738$ ) (Fig. 3, Table 4). Target lesion revascularization was performed on $4(6.7 \%)$ patients in the DES-DESolve group and 5 $(11.6 \%)$ patients in the DESolve-DESolve group $(p=0.485)$. Three patients suffered BVS thrombosis. Two patients had DESolveDESolve thrombosis on the third day and 2 months following overlapping, whereas one patient had very late DES-DESolve thrombosis at 47 months after the index procedure.

\section{Discussion}

Following the initial interest in BVSs, poor clinical results have hindered the clinical use of other BVSs concerning TLR 


\begin{tabular}{|c|c|c|c|}
\hline Variables & $\begin{array}{c}\text { DESolve-DESolve } \\
n=43\end{array}$ & $\begin{array}{c}\text { DES-DESolve } \\
n=60\end{array}$ & $P$-value \\
\hline Type A lesion & $1(2.3 \%)$ & $7(11.7 \%)$ & 0.358 \\
\hline Type B1 lesion & $14(32.6 \%)$ & $18(30 \%)$ & 0.358 \\
\hline Type B2 lesion & $19(44.2 \%)$ & $22(36.7 \%)$ & 0.358 \\
\hline Type C Iesion & $9(20.9 \%)$ & $13(21.7 \%)$ & 0.358 \\
\hline Severe tortiosis, $n$ & $7(16.3 \%)$ & $12(20 \%)$ & 0.564 \\
\hline $\begin{array}{l}\text { Severe calcification, } \\
\mathrm{n}(\%)\end{array}$ & $20(46.5 \%)$ & $26(43.3 \%)$ & 0.520 \\
\hline Lesion length, mm, & $45.86 \pm 6.4$ & $47.86 \pm 8.1$ & 0.076 \\
\hline Reference diameter & $3.01 \pm 0.41$ & $2.94 \pm 0.36$ & 0.737 \\
\hline Stent length, mm & $25.67 \pm 5.04$ & $26.87 \pm 3.7$ & 0.048 \\
\hline Stent diameter, mm & $2.98 \pm 0.4$ & $2.90 \pm 0.4$ & 0.577 \\
\hline 2. Stent length, mm & $25.76 \pm 4.2$ & $25.58 \pm 7.7$ & 0.001 \\
\hline 2. Stent diameter, mm & $3.18 \pm 0.38$ & $3.08 \pm 0.34$ & 0.167 \\
\hline Acute gain & $1.69 \pm 0.66$ & $1.63 \pm 0.71$ & 0.306 \\
\hline Pre-PCI MLD & $0.93 \pm 0.45$ & $0.81 \pm 0.54$ & 0.573 \\
\hline Post-balloon MLD & $2.02 \pm 0.37$ & $1.9 \pm 0.43$ & 0.348 \\
\hline Post-stent MLD & $2.73 \pm 0.36$ & $2.72 \pm 0.40$ & 0.514 \\
\hline \multicolumn{4}{|l|}{ Treated vessel, n (\%) } \\
\hline LAD & $32(74.4 \%)$ & $40(66.7 \%)$ & 0.694 \\
\hline $\mathrm{CX}$ & $2(4.7 \%)$ & $4(6.7 \%)$ & \\
\hline RCA & $9(20.9 \%)$ & $16(26.7 \%)$ & \\
\hline
\end{tabular}

CX - circumflex; DES - drug eluting stent; LAD - left anterior descending; MLD - Minimal lumen diameter; RCA - right coronary artery

Table 4. Clinical outcomes at 4-year follow-up

\begin{tabular}{|lccc|}
\hline & $\begin{array}{c}\text { DESolve-DESolve } \\
\mathbf{n = 4 3}\end{array}$ & $\begin{array}{c}\text { DES-DESolve } \\
\mathbf{n}=\mathbf{6 0}\end{array}$ & $\begin{array}{c}\boldsymbol{P} \text {-value } \\
\text { log-rank }\end{array}$ \\
\hline All-cause death & 0 & $1(1.7 \%)$ & 1 \\
Cardiac death & 0 & 0 & N/A \\
TV-MI & $3(7 \%)$ & $2(3.3 \%)$ & 0.396 \\
Definite ST & $1(2.3 \%)$ & $2(3.3 \%)$ & 0.764 \\
TLR & $5(11.6 \%)$ & $4(6.7 \%)$ & 0.485 \\
TVR & $5(11.6 \%)$ & $6(10 \%)$ & 0.792 \\
MACE & $5(11.6 \%)$ & $5(8.3 \%)$ & 0.738 \\
\hline
\end{tabular}

DES - drug-eluting-stent; MACE - major adverse cardiac event; TV-MI - target vessel myocardial infarction; TLR - target lesion revascularization; TVR - target vessel revascularization; ST - scaffold thrombosis; NA - not available

and the scaffold thrombosis of everolimus-eluting BVS (Absorb), which is regarded as the most extensively researched BVS (10). In 4 randomized Absorb studies that treated patients with Absorb ( $n=2164)$ or DES ( $n=1225)$, a meta-analysis resulted in increased 3-year targeted lesion failing ratios in the Absorb group (11.7\% vs. $8.1 \%$; $p=0.006)$ (11). Therefore, the application of
BVS has been limited to clinical research. Although there are some studies on long-segment Absorb BVS, there are no studies on long segment DESolve BVS (12). There are almost no studies on long-term results. We believe that our study may contribute to future studies with larger patient groups.

Early findings from 5-year DESolve Nx monitoring show no apparent scaffold thrombosis, $4(2.5 \%)$ cardiac deaths, and 5 (4.1\%) scientifically induced TLRs, respectively. Within 2 years, the MACE average was $7.4 \%$ (13). Lower-risk lesion subgroups (mean lesion distance $11.2 \pm 3.8 \mathrm{~mm}$, lesion incidence Class $\mathrm{B} 2 / \mathrm{C}$ of $34 \%$ ) were found in this study. A 1-year follow-up, multicenter, post-marketing assessment of DESolve (100 patients infected with 109 lesions) revealed no cardiac deaths, 1 confirmed scaffold thrombosis (1\%), $1 \mathrm{MI}(1 \%)$, and 3 device-oriented comparative endpoints $(3 \%)$. A rapid resorption time is defined by the DESolve unit, which reabsorbs in about one year (14). The overexpansion capabilities of DESolve are also beneficial for these lesions. A study by Verheye et al. $(15,16)$ found that a 5 -year MACE is $11(9.0 \%)$. Similarly, our study shows positive results with using DESolve scaffolds in our clinic (17).

The edge-to-edge placement of two stents without overlapping techniques is linked with a higher incidence of stent thrombosis and restenosis because of the gap risk. However, increased thrombogenicity and the development of thrombus as well as some late re-endothelialization is also linked to overlapping $(18,19)$.

In a study comparing the 2-year results of overlapped everolimus-eluting stents (EES) and resolute zotarolimus-eluiting stents (R-ZES), MACE was $5.8 \%$ for EES vs. $8.1 \%$ for R-ZES; $p=0.548$, and TVR was $3.4 \%$ for EES vs. $4.0 \%$ for R-ZES; $p=0.806$. The resulting MACE and TVR rates of this study are similar to those of our study (20).

In a report on Absorb performed on long-segment lesions with reported 12-month findings, researchers observed that when Absorb overlapping and non-overlapping were compared, there was no considerable difference in 12-month patient outcomes $(21,22)$. However, findings from another study revealed that overlapping BVS placement was an independent risk factor for peri-procedural MI (23).

In a study overlapping the porcine coronary artery with Absorb, it was confirmed that enhanced neointimal hyperplasia and prolonged stent covering leading to denser strut structure could contribute to scaffold restenosis and thrombosis (24). Another in vitro phantom research study reported a high possibility of spatial damage because of the insufficient recognition of scaffold edge markers and the difficulty of positioning the scaffold edges in the same location. It was speculated that minimizing the overlap area may mitigate the risk of undesirable patient outcomes $(25,26)$.

The optical coherence tomography (OCT) results from GHOST-CTO analysis found that neointimal hyperplasia was more commonly observed in the overlapping section in the vessel overlap area where more than one bioresorbable scaffold was included (27). 
Gil et al. (28) found that in the first analysis, Absorb-DES hybrid overlap can be successfully performed on patients with long-segment lesions with high procedural performance and low MACE and complication rates. In addition, our study found no statistical significance in the MACE ratios in Absorb-DES or Absorb-Absorb overlapping when the short-term effects of overlap lesions were analyzed in our clinic (29).

By avoiding both long-segment overlapping and gap generation, the utilization of the DESolve and the decreased overlapping technique may have reduced the risk associated with the use of BVS as well as thicker struts.

Long-term follow-ups were exclusively documented and evaluated at our clinic. Inadequate clinical outcomes for BVSs are being documented in the long run, and the hybrid overlapping of BVS-BVS and DES-BVS combined were investigated, which provided comparable results to previous studies, suggesting that both techniques could be reliably used for overlapping. Many parameters were considered for observing this condition.

We proposed to administer antiplatelet therapy for one year in patients we performed BVS implantation. However, owing to the thrombosis results reported by many centers during the follow-up, we planned to use dual antiplatelet therapy (DAPT) for more than one year, considering the risk of bleeding. We followed the patients who were given new P2Y12-receptor antagonists (ticagrelor or prasugrel) in the first year and switching to clopidogrel after the first year. On the basis of opinions on this issue, we have extended DAPT period up to 3 years by making a patient-specific decision $(30,31)$. One of the reasons for the low bleeding rates was the relatively young age of our patients.

Our analysis found MACE occurrence to be relatively low and did not detect stent thrombosis. We clarified this situation by using DESolve (32) and strictly complying with the deployment of BVS during the procedures conducted by the same surgeon. It can be speculated that the adoption of an optimal pre-dilation, appropriate sizing, and post-dilation to high pressure protocol (PSP) with growing experience over time might have influenced the reported outcomes.

\section{Study limitations}

Quantitative coronary angiography, a functional approach that provides substantial knowledge about lesion characteristics, was tested for angiographic procedural success. However, more specific details may have been generated by the use of other strategies, such as intravascular ultrasound (IVUS) or OCT. Similarly, the OCT or IVUS overlap site evaluation may further explain the existence of non-endothelialized areas under DES after BVS degradation. However, access to IVUS and OCT is not easy everywhere; therefore, this could be the most important reason for limitation.

\section{Conclusion}

Although it is not recommended to use Absorb in practice owing to its long-term results, not all BVSs should be evaluated in a similar manner. The positive results of our study concerning the use of DESolve for the treatment of long coronary lesions demonstrate that BVS will emerge with new platforms and become non-inferior to DES.

Declaration: It was presented as a poster presentation at the EUROPCR Congress held in Paris between 18-21 May 2021.

Conflict of interest: None declared.

Peer-review: Externally peer-reviewed.

Author contributions: Concept - E.I.; Design - E.l.; Supervision B.Ç.; Fundings - None; Materials - E.l.; Data collection \&/or processing - E.I., S.Ç.; Analysis \&/or interpretation - B.Boyraz; Literature search H.M.G.; Writing - E.l.; Critical review - B.Boztosun

\section{References}

1. O'Sullivan CJ, Stefanini GG, Räber L, Heg D, Taniwaki M, Kalesan B, et al. Impact of stent overlap on long-term clinical outcomes in patients treated with newer-generation drug-eluting stents. Eurolntervention 2014; 9: 1076-84. [Crossref]

2. Räber $L$, Jüni $P$, Löffel $L$, Wandel $S$, Cook $S$, Wenaweser $P$, et al. Impact of stent overlap on angiographic and long-term clinical outcome in patients undergoing drug-eluting stent implantation. $J$ Am Coll Cardiol 2010; 55: 1178-88. [Crossref]

3. Farooq V, Vranckx P, Mauri L, Cutlip DE, Belardi J, Silber S, et al. Impact of overlapping newer generation drug-eluting stents on clinical and angiographic outcomes: pooled analysis of five trials from the international Global RESOLUTE Program. Heart 2013; 99: 626-33. [Crossref]

4. Ormiston JA, Serruys PW. Bioabsorbable coronary stents. Circ Cardiovasc Interv 2009; 2: 255-60. [Crossref]

5. Serruys PW, Garcia-Garcia HM, Onuma Y. From metallic cages to transient bioresorbable scaffolds: change in paradigm of coronary revascularization in the upcoming decade? Eur Heart J 2012; 33: 16-25b. [Crossref]

6. Onuma Y, Dudek D, Thuesen L, Webster M, Nieman K, GarciaGarcia HM, et al. Five-year clinical and functional multislice computed tomography angiographic results after coronary implantation of the fully resorbable polymeric everolimus-eluting scaffold in patients with de novo coronary artery disease: the ABSORB cohort A trial. JACC Cardiovasc Interv 2013; 6: 999-1009. [Crossref]

7. Mattesini A, Bartolini S, Sorini Dini C, Valente S, Parodi G, Stolcova $M$, et al. The DESolve novolimus bioresorbable Scaffold: from bench to bedside. J Thorac Dis 2017; 9(Suppl 9): S950-8. [Crossref]

8. Ho HH, Jafary FH, Loh KK, Tan JK, Ooi YW, Ong PJ. Deliverability of integrity coronary stents in severely tortuous coronary arteries: a preliminary experience. J Invasive Cardiol 2012; 24: 650-4.

9. Mintz GS, Popma JJ, Pichard AD, Kent KM, Satler LF, Chuang YC, et al. Patterns of calcification in coronary artery disease. A statistical analysis of intravascular ultrasound and coronary angiography in 1155 lesions. Circulation 1995; 91: 1959-65. [Crossref]

10. Puricel S, Cuculi F, Weissner M, Schmermund A, Jamshidi $P$, Nyffenegger T, et al. Bioresorbable Coronary Scaffold Thrombosis: Multicenter Comprehensive Analysis of Clinical Presentation, Mechanisms, and Predictors. J Am Coll Cardiol 2016; 67: 921-31. [Crossref] 
11. Ali ZA, Gao R, Kimura T, Onuma Y, Kereiakes DJ, Ellis SG, et al. Three-Year Outcomes With the Absorb Bioresorbable Scaffold: Individual-Patient-Data Meta-Analysis From the ABSORB Randomized Trials. Circulation 2018; 137: 464-79. [Crossref]

12. Ali ZA, Serruys PW, Kimura T, Gao R, Ellis SG, Kereiakes DJ, et al. 2-year outcomes with the Absorb bioresorbable scaffold for treatment of coronary artery disease: a systematic review and metaanalysis of seven randomised trials with an individual patient data substudy. Lancet 2017; 390: 760-72. [Crossref]

13. Abizaid A, Costa RA, Schofer J, Ormiston J, Maeng M, Witzenbichler $B$, et al. Serial Multimodality Imaging and 2-Year Clinical Outcomes of the Novel DESolve Novolimus-Eluting Bioresorbable Coronary Scaffold System for the Treatment of Single De Novo Coronary Lesions. JACC Cardiovasc Interv 2016; 9: 565-74. [Crossref]

14. Nef H, Wiebe J, Boeder N, Dörr O, Bauer T, Hauptmann KE, et al. A multicenter post-marketing evaluation of the Elixir DESolve ${ }^{\circledR}$ Novolimus-eluting bioresorbable coronary scaffold system: First results from the DESolve PMCF study. Catheter Cardiovasc Interv 2018; 92: 1021-7. [Crossref]

15. Verheye S, Ormiston JA, Stewart J, Webster M, Sanidas E, Costa R, et al. A next-generation bioresorbable coronary scaffold system: from bench to first clinical evaluation: 6 - and 12-month clinical and multimodality imaging results. JACC Cardiovasc Interv 2014; 7: 89-99.[Crossref]

16. Verheye S, Costa RA, Schofer J, Ormiston JA, Maeng M, Dudek D, et al. Five-year safety and performance data of a novel third-generation novolimus-eluting bioresorbable scaffold in single de novo lesions. Eurolntervention. 2019; 15: 685-7. [Crossref]

17. Cakal B, Cakal S, Karaca O, Omaygenc MO, Yilmaz FK, Gunes HM, et al. Outcomes of the novolimus-eluting bioresorbable vascular scaffold in real world clinical practice. Minerva Cardiol Angiol 2021; 69: 261-8. [Crossref]

18. Kolandaivelu K, Swaminathan R, Gibson WJ, Kolachalama VB, Nguyen-Ehrenreich KL, Giddings VL, et al. Stent thrombogenicity early in high-risk interventional settings is driven by stent design and deployment and protected by polymer-drug coatings. Circulation 2011; 123: 1400-9. [Crossref]

19. Murasato Y, Hikichi Y, Nakamura S, Kajiya F, Iwasaki K, Kinoshita Y, et al. Recent perspective on coronary bifurcation intervention: statement of the "Bifurcation Club in KOKURA". J Interv Cardiol 2010; 23: 295-304. [Crossref]

20. Li XT, Sun H, Zhang DP, Xu L, Ni ZH, Xia K, et al. Two-year clinical outcomes of patients with overlapping second-generation drugeluting stents for treatment of long coronary artery lesions: comparison of everolimus-eluting stents with resolute zotarolimuseluting stents. Coron Artery Dis 2014; 25: 405-11. [Crossref]

21. Ortega-Paz L, Capodanno D, Giacchi G, Gori T, Nef H, Latib A, et al. Impact of overlapping on 1-year clinical outcomes in patients undergoing everolimus-eluting bioresorbable scaffolds implantation in routine clinical practice: Insights from the European multicenter GHOST-EU registry. Catheter Cardiovasc Interv 2017; 89: 812-8. [Crossref]
22. Blachutzik F, Boeder N, Wiebe J, Mattesini A, Dörr O, Most A, et al. Overlapping implantation of bioresorbable novolimus-eluting scaffolds: an observational optical coherence tomography study. Heart Vessels 2017; 32: 781-9. [Crossref]

23. Ishibashi Y, Muramatsu T, Nakatani S, Sotomi Y, Suwannasom P, Grundeken MJ, et al. Incidence and Potential Mechanism(s) of Post-Procedural Rise of Cardiac Biomarker in Patients With Coronary Artery Narrowing After Implantation of an EverolimusEluting Bioresorbable Vascular Scaffold or Everolimus-Eluting Metallic Stent. JACC Cardiovasc Interv 2015; 8: 1053-63. [Crossref]

24. Farooq V, Serruys PW, Heo JH, Gogas BD, Onuma Y, Perkins LE, et al. Intracoronary optical coherence tomography and histology of overlapping everolimus-eluting bioresorbable vascular scaffolds in a porcine coronary artery model: the potential implications for clinical practice. JACC Cardiovasc Interv 2013; 6: 523-32. [Crossref]

25. Farooq V, Onuma Y, Radu M, Okamura T, Gomez-Lara J, Brugaletta $S$, et al. Optical coherence tomography (OCT) of overlapping bioresorbable scaffolds: from benchwork to clinical application. Eurolntervention 2011; 7: 386-99. [Crossref]

26. Yew KL. Overlapping technique for hybrid percutaneous coronary intervention strategy utilising drug eluting stent and ABSORB bioresorbable vascular scaffold. Int J Cardiol 2015; 178: e8-10. [Crossref]

27. Pereira GTR, La Manna A, Ichibori $Y$, Vergara-Martel A, Ramos Nascimento B, Samdani AJ, et al. Optical coherence tomography evaluation of the absorb bioresorbable scaffold performance for overlap versus non-overlap segments in patients with coronary chronic total occlusion: insight from the GHOST-CTO registry. Int J Cardiovasc Imaging 2019; 35: 1767-76. [Crossref]

28. Gil RJ, Bil J, Pawłowski T, Yuldashev N, Kołakowski L, Jańczak J, et al. The use of bioresorbable vascular scaffold Absorb BVS ${ }^{\circledR}$ in patients with stable coronary artery disease: one-year results with special focus on the hybrid bioresorbable vascular scaffolds and drug eluting stents treatment. Kardiol Pol 2016; 74: 627-33. [Crossref]

29. Güneş HM, Gökdeniz T, Karaca I0, Günhan Demir G, Kızılırmak F, Güler E, et al. Safety and Efficacy Outcomes of Bioresorbable Scaffolds in Long Segment Coronary Lesions. Koşuyolu Heart Journal 2018; 21: 53-60. [Crossref]

30. Mauri L, Kereiakes DJ, Yeh RW, Driscoll-Shempp P, Cutlip DE, Steg PG, et al.; DAPT Study Investigators. Twelve or 30 months of dual antiplatelet therapy after drug-eluting stents. N Engl J Med 2014; 371: 2155-66. [Crossref]

31. Capodanno D, Angiolillo DJ. Antiplatelet Therapy After Implantation of Bioresorbable Vascular Scaffolds: A Review of the Published Data, Practical Recommendations, and Future Directions. JACC Cardiovasc Interv 2017; 10: 425-37. [Crossref]

32. Demola P, Meucci F, Stolcova M, Mario CD, Mattesini A. The DESolve ${ }^{\circledR}$ novolimus bioresorbable scaffold. Future Cardiol 2021; 17: 945-51. [Crossref] 